\title{
Effect of Uncertainty on Deterministic Runway Scheduling
}

\author{
Gautam Gupta $^{\mathrm{a}}$ and Waqar Malik ${ }^{\mathrm{b}}$ \\ University of California, Santa Cruz, NASA Ames Research Center, Moffett Field, CA 94035, USA \\ Yoon C Jung ${ }^{\mathrm{c}}$ \\ NASA Ames Research Center, Moffett Field, CA 94035, USA
}

\begin{abstract}
Active runway scheduling involves scheduling departures for takeoffs and arrivals for runway crossing subject to numerous constraints. This paper evaluates the effect of uncertainty on a deterministic runway scheduler. The evaluation is done against a firstcome-first-serve scheme. In particular, the sequence from a deterministic scheduler is frozen and the times adjusted to satisfy all separation criteria; this approach is tested against FCFS. The comparison is done for both system performance (throughput and system delay) and predictability, and varying levels of congestion are considered. The modeling of uncertainty is done in two ways: as equal uncertainty in availability at the runway as for all aircraft, and as increasing uncertainty for later aircraft. Results indicate that the deterministic approach consistently performs better than first-come-first-serve in both system performance and predictability.
\end{abstract}

\section{Introduction}

Active runway scheduling involves scheduling departures for takeoffs and arrivals for runway crossing subject to numerous constraints. These constraints include wake separation constraints for successive departures, miles-in-trail separation for aircraft bound for the same departure fixes, and time-window or prioritization constraints for individual flights. Runways have been identified as the main source of airport delay in existing literature ${ }^{1}$, and runway scheduling has been an active field for research. Runway scheduling is also a critical component in other airport optimization schemes, such as the metering of departures ${ }^{2}$ which has been shown to provide significant benefits at an airport ${ }^{3}$. However, a majority of these are deterministic schedulers which do not incorporate the uncertainty in aircraft push back and taxiing.

The runway scheduling problem has been treated as an example of the generic job-shop scheduling problem and a dynamic programming algorithm for single machine scheduling was developed and applied to aircraft landing ${ }^{4}$. Other papers also address the aircraft landing problem as a job-shop scheduling problem ${ }^{5-7}$ using a mixed integer formulation. Constraint Position Shifting (CPS) has been proposed for the dynamic scheduling of arrival aircraft along with heuristics to solve it $^{8}$. The idea of CPS was further incorporated into a dynamic programming approach for scheduling aircraft landings ${ }^{9}$ and was later extended to departure scheduling ${ }^{10}$. CPS reduces the solution space by limiting the number of positions an aircraft can occupy in the sequence and thus could lead to suboptimal solutions. The dynamic programming approach (see Ref. 10) has been extended into a generalized dynamic program for solving the departure scheduling problem ${ }^{11}$. This method determines pareto-optimal solutions for multiple objectives but does not assign aircraft to available "queues" (when the taxiway area next to a departure runway is operated as queues). In a recent paper $^{12}$, the authors determine runway queues for individual departure aircraft and schedule them within the same framework, with the framework capable of handling both with and without queue situations. This included the arrival scheduling within the decision process.

The literature mentioned above pertains to runway operations planning for the deterministic case, and does not address the inherent uncertainty in the process due to uncertainty in aircraft movement. Although stochastic methods for addressing runway operations planning have been developed ${ }^{13}$, there is a gap in testing the benefits of the deterministic schedulers in the presence of uncertainty. This paper attempts to fill that gap by testing a deterministic scheduler under uncertainty, and a large spectrum of possibilities (including different traffic densities,

\footnotetext{
${ }^{a}$ Associate Research Scientist, UARC, Building 210, MS 210-8, Moffett Field, CA-94035. ggupta@ucsc.edu.

${ }^{\mathrm{b}}$ Associate Research Scientist, UARC, Building 210, MS 210-8, Moffett Field, CA-94035. wmalik@ucsc.edu.

${ }^{c}$ Aerospace Engineer, NASA Ames Research Center, MS 210-6, Moffett Field, CA 94035. yoon.c.jung@nasa.gov.
} 
aircraft mix, presence of miles-in-trail separation constraints, varying values of uncertainty) are considered. The uncertainty modeled here stems from uncertainty in aircraft push back and taxiing, and is modeled as uncertainty in earliest readiness for take-off or arrival crossing. The performance under uncertainty is evaluated as the relative merit over first-come-first serve (FCFS) for metrics of benefits and predictability. In particular, the sequence from a deterministic scheduler is frozen and the times were adjusted to satisfy all separation criteria; this approach is tested against FCFS. Such tests of performance under uncertainty give insights into the models' (and optimization schemes') use in a real world situation, as well as preliminary benchmarks for benefits realized under a particular "level of uncertainty."

The rest of the document is organized as follows: the next section discusses the approach and the deterministic scheduler used for the tests. This includes the setup of the experiments and the test parameters, as well as the metric used for comparison. Section III and IV shows results for different parameters, and section V concludes with directions for future work.

\section{Approach}

For the purpose of testing under uncertainty the approach developed in Ref. 12 is used, which is based on mixed integer linear programming. The approach can address a variety of queue configurations and thus allows for testing a variety of scenarios. The mixed integer linear program (MILP) takes as input the sequence of departure (and arrival) aircraft for a runway, along with their earliest take-off (and crossing) times and an optional prioritization scheme based on a time-window of take-off (and crossing) for each aircraft. The model then assigns departure aircraft to the available departure queues and schedules runway usage times for both departure and arrival crossings. For the ease of referencing, the model and its explanation have been reproduced below in Section A:

\section{A. Basic Mathematical Formulation}

Consider a set of flights $F$, which represents the aircraft expected to use the departure runway (either take-off or crossing) within the planning horizon. Let $A$ and $D$ be two subsets of $F$, denoting arrival flights and departing flights respectively. Each member of the set $D$ will have an aircraft type and destination departure fix, which would govern the separation from preceding and succeeding aircraft. Each member of $A$ will have an aircraft type and the assigned crossing. Further, each aircraft in $F$ will have a time of earliest runway usage based on when it enters the queuing area (un-impeded, or assuming no other aircraft is present) or is available to cross. Let this time be $a_{f}$ for flight $f(f \in F)$, and let the set $F$ be sorted in ascending order of $a_{f}$. In other words, if $i, j \in F$ then $i>j$ implies $a_{i}>a_{j}$. Each aircraft may have a window of runway usage, and let us denote this with $\Delta_{f}$. This time window could be strict (that the aircraft has to depart before $a_{f}+\Delta_{f}$ ) in which case smaller values of $\Delta_{f}$ could lead to infeasibility, or the window could be lenient, with a penalty for violation. In the formulation presented here the time window is modeled as a strict constraint. With the small modification, this formulation can be changed to accommodate lenient, penalized time-windows too. These windows can be used as a means to prioritize some aircraft. Of course, prioritization is optional and the windows could be sufficiently large to avoid any prioritization. Let $P$ denote the set of positions these aircraft can occupy in the "usage sequence" from the runway. Thus, $|F|=|P|$, or the number of such positions in the output sequence are equal to the input number of aircraft. The rest of this section describes the notation, summarizing the above set of parameters and describing the variables. This is followed by the complete mathematical formulation of the MILP and an explanation of all the expressions.

\section{Parameters}

$F \quad$ is the set of all incoming flights, sorted in ascending order of earliest un-impeded time at runway

$A \quad$ is the set of all arrival flights, $A \subset F$

$D \quad$ is the set of all departure flights, $D \subset F$

$P \quad$ is the set of positions that flights can take in the departure sequence on the runway, $|F|=|P|$

$Q \quad$ is the set of all departure queues that the departure aircraft can be assigned to

$C \quad$ is the set of all crossings for the runway, and each arrival flight is on one of these crossings

$a_{f} \quad$ is the earliest runway usage time for flight $f(f \in F)$

$\Delta_{f} \quad$ is the time window of runway usage for flight $f(f \in F)$ 
$h_{f, c} \quad$ is the additional waiting time before arrival flight $f$ crosses due to the usage of crossing $c,(f \in A, c \in C)$

$w_{f, c} \quad$ is a binary parameter, and is 1 if $c$ is the crossing for arrival flight $f$, zero otherwise $(f \in A, c \in C)$

$d_{i, j} \quad$ is the minimum separation in runway usage times of flight $i$ and $j$, if flight $i$ follows flight $j(i, j \in F$ ) on the runway. This depends on a variety of factors, as described below.

The parameter $d_{i, j}$ defines the required separation between the two aircraft, and depends on whether the aircraft are arrivals or departures, and in the case of arrivals, the assigned arrival crossing. Let

$\boldsymbol{e}_{i, j}^{1} \quad$ be the required separation in runway usage times of departure flights $i$ and $j$, if flight $i$ follows flight $j(i, j \in D)$ on the runway.

$e_{i, j}^{2} \quad$ be the required separation in runway usage times of departure flight $i$ and arrival flight $j$, if flight $i$ follows flight $j(i \in D, j \in A)$ on the runway

$e_{i, j}^{3} \quad$ be the required separation in runway usage times of arrival flight $i$ and departure flight $j$, if flight $i$ follows flight $j(i \in A, j \in D)$ on the runway

$g_{c} \quad$ be the required separation between two arrival flights when both are assigned to crossing $c,(c \in C)$

Note that $e_{i, j}^{1}$ is the minimum separation between the two departure aircraft considering all the separation criteria including wake-vortex separation, miles-in-trail restriction etc. Further, depending on the assigned crossing, there could be additional delay $\left(h_{f, c}\right)$. Incorporating all these cases, the parameter $d_{i, j}$ is defined as below:

$$
d_{i, j}=\left\{\begin{array}{cc}
e_{i, j}^{1} & (i, j \in D) \\
e_{i, j}^{2} & (i \in D, j \in A) \\
e_{i, j}^{3}+\sum_{c \in C} w_{i, c} h_{i, c} & (i \in A, j \in D) \\
g_{c}\left(\left(\sum_{c \in C} w_{i, c} h_{i, c}-\sum_{c \in C} w_{j, c} h_{j, c}\right), 0\right) & \left(i, j \in A, w_{i, c}=w_{j, c}=1, c \in C\right) \\
\max \left(i, j \in A, w_{i, c}+w_{j, c} \leq 1, c \in C\right)
\end{array}\right.
$$

\section{Variables}

$y_{f, p} \quad$ is a binary variable, and is 1 if flight $f(f \in F)$ occupies position $p(p \in P)$, zero otherwise

$x_{f, q} \quad$ is a binary variable, and is 1 if departure flight $f(f \in D)$ is assigned to queue $q(q \in Q)$, zero otherwise

$t_{p} \quad$ is the runway usage time of aircraft assigned to position $p$

\section{Mathematical Formulation}

$$
\begin{gathered}
\text { Minimize System Delay: minimize } \sum_{p \in P} t_{p}-\sum_{f \in F} a_{f} \\
\text { Maximize Throughput: minimize } t_{|P|} \\
\text { Minimize Maximum Delay: minimize } \max _{p \in P}\left(t_{p}-\sum_{f \in F} y_{f, p} a_{f}\right)
\end{gathered}
$$

such that

$$
\sum_{p \in P} y_{f, p}=1 \quad \forall f \in F
$$




$$
\begin{gathered}
\sum_{f \in F} y_{f, p}=1 \quad \forall p \in P \\
\sum_{q \in Q} x_{f, q}=1 \quad \forall f \in D \\
t_{p} \geq \sum_{f \in F} y_{f, p} a_{f} \quad \forall p \in P \\
t_{p} \leq \sum_{f \in F} y_{f, p}\left(a_{f}+\Delta_{f}\right) \quad \forall p \in P \\
\sum_{p} \geq t_{p^{\prime}}+\left(y_{i, p}+y_{j, p^{\prime}}-1\right) d_{i, j} \quad \forall i, j \in F, p, p^{\prime} \in P, p>p^{\prime} \\
\left.\sum_{u \in P}\right) u-\sum_{v \in P}\left(y_{j, v}\right) v \geq|P|\left(x_{i, q}+x_{j, q}-2\right) \quad \forall q \in Q, i, j \in D, i>j \\
\sum_{u \in P}\left(y_{i, u}\right) u \geq \sum_{v \in P}\left(y_{j, v}\right) v \quad w_{i, c}=w_{j, c}=1, c \in C, i>j, i, j \in D
\end{gathered}
$$

As stated before, each departure aircraft is assigned to a departure queue and then assigned a departure time. The time assignment is done by first assigning each aircraft a position in the output departure sequence (if it is a departure aircraft), and then maintaining the separation between consecutive positions. The expressions (2) to (11) can be interpreted as follows:

Eq. (2), (3) and (4) are the three objective functions labeled according to the pertinent objective. Eq. (2) is the system delay objective, and minimizes the total time spent by each aircraft in departure queue (or crossing for arrivals) beyond the earliest, unimpeded runway usage time. Eq. (3) is the throughput objective that minimizes the take-off time of the last position $t_{|p|}$. Eq. (4) is the maximum delay objective, which minimizes the maximum time spent by any aircraft in the queue area.

Constraint (5) mandates that each aircraft will occupy just one position in the output sequence.

Constraint (6) is similar to (5), and represents the fact that each position in the output sequence must be occupied by just one aircraft.

Constraint (7) ensures that each departure aircraft can occupy just one departure queue.

Constraints (8) and (9) ensure that if aircraft $f$ is assigned to position $p$, then the runway usage time for position $p$ is within the time window of aircraft $f$.

Constraint (10) ensures that the runway usage times for any two positions are sufficiently spaced out to ensure the separation criteria flights assigned to the positions. If flight $i$ is assigned to position $p\left(y_{i, p}=1\right)$ and flight $\mathrm{j}$ is assigned to position $p\left(y_{i, p}=1\right)$ and flight $j$ is assigned to position $-k\left(y_{j, p-k}=1\right)$, then $t_{p} \geq t_{p-k}+d_{i, j}$, otherwise the constraint is "inactive," or dominated by the constraint for the pair of aircraft that are assigned to these positions.

Constraint (11) ensures the first-in-first-out (FIFO) structure of each departure queue. The two terms on the left hand side of the inequality give the difference in the position of aircraft $i$ and $(i>j)$. If both the aircraft are assigned to the same queue $\left(x_{i, q}=x_{j, q}=1\right)$, then the difference in the positions should be at least one, with $i$ occupying a position after $j$ since $i$ entered the queue later than $j$. Similarly, constraint (12) ensures the FIFO structure of the arrival crossings.

To address scenarios with pre-determined departure queues, additional constraints can be included in the problem, such as $x_{f, q}=1$ for the relevant queue and departure flight. The rest of the formulation remains the same. In fact, for these scenarios, $x_{f, q}$ is no longer a variable but an input parameter, and thus the problem size is reduced.

For scenarios with no FIFO queues, essentially there is no departure queuing structure. This can be modeled by eliminating the queuing variables $x_{f, q}$ and related constraints (7) and (11) from the formulation. The reduced problem will have fewer variables and constraints, and depending on the problem characteristics, it is possible that the reduced problem is computationally more efficient.

As stated before, the above formulation models the time window as a strict constraint. This formulation can be changed to accommodate "soft" time-windows by defining an additional linear variable $s_{p}$ and adding constraints 
$s_{p} \geq t_{p}-\sum_{f} y_{f, p}\left(a_{f}+\Delta_{f}\right)$ and $s_{p} \geq 0$. Then $s_{p}$ can be multiplied by an appropriate cost of violating time windows and added to the objective function.

\section{B. Problem Setup and Objective Function}

For the purpose of testing, the above MILP was used for the "no queue" case. As is evident from the formulation, queue assignment reduces the solution space through more constraints. A no queue problem would show more changes in solutions under the presence of uncertainty in earliest runway usage times, and hence provide a larger picture of the effect of uncertainty. The effect of uncertainty in the presence of queues can also be tested; however, in this paper we limit our focus to the "no queue" case.

In Ref. 12, the authors compare the effect of using either throughput (makespan) or system delay as the objective for the runway scheduler. Over numerous runs, it is shown that using a throughput optimal solution can result in almost $150 \%$ deviation in system delay as compared to the optimal system delay value. Part of the reason for this variation could be numerous throughput optimal solutions with identical throughput values but varying sequences and aircraft times. To address this, we use the Pareto optimal solutions for throughput and system delay. When a throughput optimal solution is sought, the MILP is run twice: First, the MILP is run to find the optimal throughput objective value. Then, the second run, the MILP is run for optimal system delay value constraining the throughput to be the value found in the first run. Similarly, if an optimal system delay solution is sought, stage 1 includes running for optimal system delay, and stage 2 involves running for optimal throughput while constraining the system delay to be the value found in stage 1 .

For the results in this paper, the effect of using either throughput or system delay as the planning objective are presented.

\section{Metrics for Comparison}

To test the effect of uncertainty on a deterministic runway scheduler, we generated random problem sets of various sizes under various levels of uncertainty. The problem sets were generated based on probability distributions, which are explained further in Section D. For each such problem, earliest runway usage times ( $a_{f}$, earliest runway usage time for flight $f$ ) are known. Henceforth, we call this the "Un-Perturbed" problem. The $a_{f}$ values are then perturbed based on assumed distribution of uncertainty $\left(\theta_{f}\right.$ be the perturbation in $a_{f}$, with $a_{f}+\theta_{f}$ being the new earliest arrival times). We call this the "Perturbed" problem.

Essentially, two different approaches are compared here:

i. FCFS approach, where each aircraft is served in the order they are available. In this case, a FCFS solution is expected for the un-perturbed problem, and a different FCFS solution may be realized for the perturbed problem.

ii. Deterministic Sequence (DS) approach, where a deterministic solution is generated based on the unperturbed problem, and in the presence of uncertainty the sequence from the deterministic solution is frozen and times adjusted to get the result under uncertainty.

For each problem set, both FCFS and DS approach are explored, and the following problems are solved for analysis:

- FCFS on the Un-Perturbed problem using $a_{f}$ values to do FCFS. Let this solution be FCFS-U. This solution can be likened to what would happen if all aircraft came to the runway at their $a_{f}$ times and were served in a first-come-first-served manner.

- FCFS on the Perturbed problem using $a_{f}+\theta_{f}$ values to do FCFS. Let this solution be FCFS-P. This represents the realized FCFS based on $a_{f}+\theta_{f}$ values.

- MILP for a given objective (throughput or system delay) on Un-Perturbed problem using $a_{f}$ values. Let this solution be DS-U.

- Fixing the sequence for all aircraft to the OPT-U solution and using the perturbed times $\left(a_{f}+\theta_{f}\right)$ to change actual runway usage times to meet the separation constraints. This represents the realization of the deterministic schedule planned at the beginning, with uncertainty handled by only changing the times. Let this solution be DS-COUS (Constrained Optimal Un-perturbed Solution).

- MILP for the same objective as OPT-U on Perturbed problem using $a_{f}+\theta_{f}$. Let this solution be OPT. This represents the hindsight solution and represents the best that can be done with complete information of the future.

The FCFS approach and the DS approach are compared for system performance (throughput and system delay) as well as for predictability. The following metrics are defined for comparison: 


\section{System Performance Metrics}

System performance is measured as the relative benefit in throughput or system delay. It should be noted that there are two ways to define throughput: first, as the number of aircraft that can be served in a particular amount of time; and second, as the time required to serve a particular number of aircraft. In our case, we use the latter definition of throughput.

If throughput is chosen as the objective, for FCFS this is defined as the difference in throughput from FCFS-P and the value from OPT-P, and for the DS it is defined as the difference in throughput from COUS and from OPT-P. Let $T H R^{F C F S-P}$ denote the throughput for the FCFS-P solution (and similarly for other solutions like $T H R^{D S-C O U S}$ etc.) Then, the metrics for system performance when throughput is chosen as the objective are:

$$
\begin{aligned}
& \text { FCFS throughput metric }=T H R^{F C F S-P}-T H R^{O P T} \\
& \text { DS throughput metric }=T H R^{D S-C O U S}-T H R^{O P T}
\end{aligned}
$$

This shows the relative benefit of FCFS and DS as "how far away they are from hindsight solution." Further, comparing these two values shows the relative merit of DS over FCFS.

Similar to the above, if system delay is chosen as the objective and $S Y S^{F C F S-P}$ denotes the system delay from the FCFS-P solution (and SYS $S^{D S-C O U S}$ denotes the system delay for DS-COUS case and so on), then the system delay metrics are:

$$
\begin{aligned}
& \text { FCFS system delay metric }=S Y S^{F C F S-P}-S Y S^{O P T} \\
& \text { DS system delay metric }=S Y S^{D S-C O U S}-S Y S^{O P T}
\end{aligned}
$$

It should be noted that all the metrics defined above are always going to be positive, since OPT represents the best possible solution, and both FCFS and DS can at best be the same.

\section{Predictability Metrics}

For predictability, we compare the individual aircraft schedule itself. Let $t_{f}$ be the actual runway usage time for flight $f$ in particular solution. Thus, $t_{f}^{D S-C O U S}$ represents the runway usage time for flight $f$ for the DS-COUS

solution, and $t_{f}^{F C F S-P}$ represents the runway usage time for flight $f$ for the FCFS-P solution. We define time predictability metric as:

$$
\begin{gathered}
\text { FCFS time predictability }=\sum_{f}\left|t_{f}^{F C F S-U}-t_{f}^{F C F S-P}\right| \\
\text { DS time predictability }=\sum_{f}\left|t_{f}^{D S-U}-t_{f}^{D S-\operatorname{COUS}}\right|
\end{gathered}
$$

The above metrics show the total variance in the initially estimated times for all aircraft and the realized times under uncertainty; this is done for both the cases: when scheduling is done based on a FCFS rule, and when scheduling is done using a deterministic schedule and under uncertainty; only the aircraft times are changed.

\section{Experimental Setup and Parameters}

The results presented in the next section are based on numerous randomly generated problem sets. The different problem sets are for scheduling an increasing number of aircraft over 15 minutes (900 seconds).

The following assumptions were made for generating these problem sets:

- Varying congestion levels (and problem sizes) are tested by scheduling 15, 16 17, 18, 19, 20 or 21 aircraft. For any problem set, the earliest runway usage times $\left(a_{f}\right)$ are generated using a uniform distribution between 0 and 900 seconds, representing increasing congestion levels over 15 minutes.

- For any problem size, there are 4 possible departure aircraft weight class ${ }^{12}$. Two fractions of arrival aircraft are tested $(30 \%$ and $50 \%)$. The remaining aircraft were identified as departures and it is assumed that all weight class have the same percentage of aircraft. For example, if one problem set has $30 \%$ arrivals, it will $(100-30) / 4=17.5 \%$ of each departure weight class.

- The required separation between different weight class and arrivals is given below in Table 1, and is based on recent human-in-the-loop experiements ${ }^{3}$.

\section{Table 1: Aircraft Types and Minimum Departure Separation (in seconds)}




\begin{tabular}{|c|c|c|c|c|c|c|c|}
\hline & & & \multicolumn{5}{|c|}{ Leading Aircraft Type } \\
\hline & & & \multicolumn{4}{|c|}{$\begin{array}{l}\text { Departure Aircraft } \\
\text { (by Type) }\end{array}$} & \multirow{2}{*}{$\begin{array}{c}\text { Arrival } \\
\text { Aircraft } \\
\text { Crossing }\end{array}$} \\
\hline & & & Small & Large & Heavy & B-757 & \\
\hline \multirow{5}{*}{$\begin{array}{c}\text { Trailing } \\
\text { Aircraft } \\
\text { Type }\end{array}$} & \multirow{4}{*}{$\begin{array}{l}\text { Departure } \\
\text { Aircraft } \\
\text { (by Type) }\end{array}$} & Small & 44 & 66 & 82 & 82 & 21 \\
\hline & & Large & 44 & 44 & 82 & 66 & 21 \\
\hline & & Heavy & 44 & 44 & 66 & 66 & 21 \\
\hline & & B 757 & 44 & 44 & 82 & 66 & 21 \\
\hline & Arrival Aircraft & Crossing & 46 & 46 & 46 & 46 & 6 \\
\hline
\end{tabular}

- Each departure aircraft is assigned one of six departure fixes, with all fixes having the same fraction of departure aircraft. Two cases are considered: one where there are no miles-in-trail (MIT) restrictions and one in which one fix has a MIT restriction of 300 seconds. This means that successive aircraft going to that fix need to be separated by at least 300 seconds.

- Two different methods are used to generate the perturbations $\left(\theta_{f}\right)$ :

- Equal perturbation window, where an equal perturbation window is defined for all aircraft. This perturbation can be negative or positive (implying late or early availability), and is uniformly distributed over this window. For example, a perturbation window of 10 seconds implies that all aircraft will have $\theta_{f}$ values based on a uniform distribution between $[-5,+5]$. Four different window sizes were considered here: 10 seconds, 30 seconds, 60 seconds and 90 seconds.

- Increasing perturbation window, where the window size is based on the $a_{f}$ value for that flight. This is to simulate the case that there is larger uncertainty in aircraft coming later to the runway. Here, the perturbation for aircraft $f$ is generated from a uniform distribution between $\left[-a_{f} / 90,+a_{f} / 90\right]$.

- For the case with an equal perturbation window, 100 different problem sets were generated for each problem size, arrival percentage, MIT restriction and perturbation size.

- For the case with an increasing perturbation size, 1000 different problem sets were generated for each problem size, arrival percentage and MIT restriction.

\section{Results for Equal Perturbation}

This section presents the results for the case when each aircraft has the same perturbation size. As stated before, four perturbation sizes are considered. The results are split into 2 parts: first - using throughput as the objective function, and second - using system delay as the objective function.

\section{A. Using Throughput (makespan) as the Objective}

\section{System Performance Metrics}

Figure 1 gives the throughput difference between FCFS-P and OPT solution (the throughput metric for FCFS approach) and between DS-COUS and OPT solution (throughput metric for DS approach). The column size represents the mean, and the $90^{\text {th }}$ and $10^{\text {th }}$ percentile are marked using the lines on each bar. All four perturbation sizes are compared for all seven congestion levels, both with and without MIT restrictions. 
Note: In each graph, the $\mathrm{x}$ axis represents varying number of aircraft (congestion) and the $\mathrm{y}$ axis shows the throughput metric (measured as difference) in seconds. Each column represents the mean and the lines on columns represent the $90^{\text {th }}$ and $10^{\text {th }}$ percentile over 100 different problem sets.

Figure 1: Throughput metric comparison between FCFS and DS approach for equal uncertainty

The following trends can be observed in the graphs: 
- The benefits of the DS approach over FCFS increase with increasing problem size, but decrease with increasing uncertainty.

- DS performs better than FCFS both in the absence and presence of MIT, but DS shows very little degradation in the presence of MIT, as compared to FCFS.

- Increasing number of arrival aircraft decreases the gap between the DS and FCFS approach. This is expected, because if a large number of aircraft are of the same type, there is little improvement by resequencing.

- The "spread" of FCFS solutions is much larger than DS solutions, showing that the performance of FCFS is very much problem-dependent, whereas that of DS is consistent and relatively independent.

\section{Predictability Metrics}

Figure 2 gives the time predictability metric for FCFS approach and DS approach as defined in equations (17) and (18) respectively. Similar to the system performance metrics, the bar size represents the mean and the $90^{\text {th }}$ and $10^{\text {th }}$ percentile are marked using the lines on each bar. All four perturbation sizes are compared for all seven congestion levels, both with and without MIT restrictions. 
Note: In each graph, the $\mathrm{x}$ axis represents varying number of aircraft (congestion) and the $\mathrm{y}$ axis shows the predictability metric in seconds. Each column represents the mean and the lines on columns represent the $90^{\text {th }}$ and $10^{\text {th }}$ percentile over 100 different problem sets.

Figure 2: Predictability (in seconds) for FCFS and DS approach for the throughput case with equal uncertainty

The following trends can be observed in the graphs:

- The predictability of DS solution is consistently better than that of the FCFS solution, and the variation of the predictability of the DS solution is also less than that of the FCFS solution.

- Predictability worsens with increasing problem size and uncertainty level for both FCFS and DS, but the trend for worsening is much steeper for FCFS.

- The introduction of MIT restriction seems to have little impact on the mean and "spread" of predictability of the DS solutions, but seems to worsen the same for the FCFS solution; however, the effect is small.

\section{B. Using System Delay as the Objective}

\section{System Performance Metrics}

Figure 3 gives the throughput difference between FCFS-P and OPT solution (FCFS approach) and between DSCOUS and OPT solution (DS approach). As before, the column size represents the mean, and the $90^{\text {th }}$ and $10^{\text {th }}$ percentile are marked using the lines on each bar. All four perturbation sizes are compared for all seven congestion levels, both with and without MIT restrictions. 
Note: In each graph, the $x$ axis represents varying number of aircraft (congestion) and the $y$ axis shows the system delay metric (measured as difference) in seconds. Each column represents the mean and the lines on columns represent the $90^{\text {th }}$ and $10^{\text {th }}$ percentile over 100 different problem sets.

Figure 3: System delay metric comparison between FCFS and DS approach for equal uncertainty

The following trends can be observed in the graphs:

- There is more variation in both the FCFS and DS solutions for system delay as compared to throughput.

- The differences between the no MIT and 300s MIT are more pronounced in the system delay case as compared to the throughput case.

- Similar to the throughput case:

- The benefits of the DS approach over FCFS increase with increasing problem size, but decrease with increasing uncertainty.

- DS performs better than FCFS both in the absence and presence of MIT, but DS shows very little degradation in the presence of MIT, as compared to FCFS.

- Increasing number of arrival aircraft decreases the gap between the DS and FCFS approach.

- The "spread" of FCFS solutions is much larger than DS solutions, showing that the performance of FCFS is very much problem-dependent, whereas that of DS is consistent and relatively independent.

11

American Institute of Aeronautics and Astronautics 


\section{Predictability Metrics}

Figure 4 gives the time predictability metric for the FCFS approach and the DS approach for system delay.

Figure 4 (a)

Figure 4 (b)

Figure 4 (c)

Figure 4 (d)

Figure $4(\mathrm{e})$

Figure 4 (f)

12

American Institute of Aeronautics and Astronautics 
Note: In each graph, the $\mathrm{x}$ axis represents varying number of aircraft (congestion) and the $\mathrm{y}$ axis shows the predictability metric in seconds. Each column represents the mean and the lines on columns represent the $90^{\text {th }}$ and $10^{\text {th }}$ percentile over 100 different problem sets.

Figure 4: Predictability (in seconds) for FCFS and DS approach for the system delay case with equal uncertainty

In predictability, the behavior of the DS approach system delay is very similar to that of the DS approach for the throughput objective:

- The predictability of the DS solution is consistently better than that of the FCFS solution, and the variation in predictability of is also less for the DS solution.

- The predictability of both DS and FCFS solution worsens with increasing problem size and uncertainty level. The introduction of MIT restriction causes a small albeit negative change in the predictability of the FCFS solution, and almost no change for the DS solution.

\section{Results for Increasing Perturbation Size}

This section presents the results for the case when an aircraft's perturbation size depends on its earliest time for runway usage. As before, the results are split into 2 parts: first - using throughput as the objective function, and second - using system delay as the objective function.

\section{A. Using Throughput as Objective}

\section{System Performance Metrics}

As shown in Figure 5, the trends observed here are similar to the case with equal uncertainty, with the DS approach consistently performing better than FCFS and the benefit increasing with increasing aircraft density. Further, DS dhows very little degradation with the inclusion of the MIT restrictions as compared to the FCFS approach.

Note: In each graph, the $x$ axis represents varying number of aircraft (congestion) and the $y$ axis shows the throughput metric (measured as difference)in seconds. Each column represents the mean and the lines on columns represent the $90^{\text {th }}$ and $10^{\text {th }}$ percentile over 100 different problem sets.

Figure 5: Throughput metric comparison between FCFS and DS approach for increasing uncertainty 


\section{Predictability Metrics}

As shown in Figure 6, even for predictability the results are similar to the case with equal window sizes with DS performing better than FCFS.

Figure $6(a)$

Figure $6(b)$

Note: In each graph, the $\mathrm{x}$ axis represents varying number of aircraft (congestion) and the $\mathrm{y}$ axis shows the predictability metric in seconds. Each column represents the mean and the lines on columns represent the $90^{\text {th }}$ and $10^{\text {th }}$ percentile over 100 different problem sets.

Figure 6: Predictability (in seconds) for FCFS and DS approach for the throughput case with increasing uncertainty

\section{B. Using System Delay as Objective}

System Performance Metrics

As shown in Figure 7, the results for this case are very similar to the case with equal perturbation size, with DS performing better than FCFS. The difference in the approaches increases with increasing problem size, and also with the inclusion of MIT restrictions.

Note: In each graph, the $x$ axis represents varying number of aircraft (congestion) and the $y$ axis shows the system delay metric (measured as difference) in seconds. Each column represents the mean and the lines on columns represent the $90^{\text {th }}$ and $10^{\text {th }}$ percentile over 100 different problem sets.

\section{Figure 7: System delay metric comparison between FCFS and DS approach for increasing uncertainty}

\section{Predictability Metrics}

As shown in Figure 8, even in predictability DS performs better than FCFS, with the difference increasing with increasing problem size. As before, inclusion of MIT restrictions makes degrades FCFS predictability, although the effect is very small. 
Note: In each graph, the $x$ axis represents varying number of aircraft (congestion) and the $y$ axis shows the predictability metric in seconds. Each column represents the mean and the lines on columns represent the $90^{\text {th }}$ and $10^{\text {th }}$ percentile over 100 different problem sets.

Figure 8: Predictability (in seconds) for FCFS and DS approach for the system delay case with increasing uncertainty

\section{Conclusions}

This paper tests the effect of uncertainty on a deterministic scheduler. In particular, the sequence from a deterministic scheduler is frozen and the times adjusted to satisfy all separation criteria; this approach is tested against FCFS. The comparison has been done for two different ways of modeling uncertainty: equal uncertainty in all aircraft or more uncertainty in later aircraft. The comparison has been done using throughput or system delay as the objective, and both system performance and predictability are compared. Different congestion levels were considered and the effect of including miles-in-trail restrictions for a departure fix was also tested.

In terms of system performance (throughput and system delay), the following trends were observed:

- For both the throughput and system delay objective DS shows more benefits than FCFS. The benefits of the DS approach over FCFS increase with increasing problem size. For the case with equal uncertainty in all aircraft, the benefits decrease with increasing uncertainty window.

- Increasing number of arrival aircraft decreases the gap between the DS and FCFS approach. This is expected, because if a large number of aircraft are of the same type, there is little improvement by resequencing.

- The "spread" of FCFS solutions is much larger than DS solutions, showing that the performance of FCFS is very much problem-dependent, whereas that of DS is consistent and relatively independent.

- DS performs better than FCFS both in the absence and presence of MIT, but DS shows very little degradation in the presence of MIT, as compared to FCFS. This is observed for both system delay and throughput. However, the differences between no MIT and 300s MIT are more pronounced in the system delay case as compared to the throughput case.

- There is more variation in both the FCFS and DS solutions for system delay as compared to throughput.

In terms of predictability of the DS and FCFS approach, the following trends were observed:

- The predictability of DS solution is consistently better than that of the FCFS solution, and the variation of the predictability of the DS solution is also less than that of the FCFS solution. This is true for with either throughput or system delay as objective.

- Predictability worsens with increasing problem for both FCFS and DS, but the trend for worsening is much steeper for FCFS. Predictability also worsens with increasing uncertainty window when there is equal uncertainty in all aircraft.

- The introduction of MIT restriction seems to have little impact on the mean and "spread" of predictability of the DS solutions, but seems to worsen the same for the FCFS solution; however, the effect is small.

The paper tests the performance under uncertainty based on a uniform distribution; similar analysis using different probability distributions is a direction for future work. Also, the version of the departure scheduler considered in this paper is the case where there are no queues. The behavior in the presence of such queues also needs to be explored. 


\section{References}

${ }^{1}$ Idris, H., Delcaire, B., Anagnostakis, I., Hall, W., Pujet, N., Feron, E., Hansman, R., Clarke, J., and Odoni, A. "Identification of flow constraint and control points in departure operations at airport systems," Air Traffic Control Quarterly Vol. 7, No. 4, 2000,

${ }^{2}$ Malik, W. A., Gupta, G., and Jung, Y. "Managing departure aircraft release for efficient airport surface operations," AIAA Guidance, Navigation, and Control Conference, Toronto, Canada, August 2-5, 2010.

${ }^{3}$ Jung, Y., Hoang, T., Montoya, J., Gupta, G., Malik, W., and Tobias, L. "A Concept and Implementation of Optimized Operations of Airport Surface Traffic " 10th AIAA Aviation Technology, Integration, and Operations Conference (ATIO), For Worth, Texas, USA, September 13-15, 2010.

${ }^{4}$ Psaraftis, H. N. "A Dynamic Programming Approach for Sequencing Groups of Identical Jobs," Operations Research Vol. 28, No. 6, 1980, pp. 1347-1359

${ }^{5}$ Bianco, L., and Bielli, M. "System aspects and optimization models in ATC planning," Large Scale Computation and Information Processing in Air Traffic Control Springer-Verlag, Berlin, 1993, pp. 47-99.

${ }^{6}$ Bianco, L., Ricciardelli, S., Rinaldi, G., and Sassano, A. "Scheduling tasks with sequence-dependent processing times," Naval Research Logistics Vol. 35, No. 2, 1988, pp. 177-184

${ }^{7}$ Bianco, L., Rinaldi, G., and Sassano, A. "A combinatorial optimization approach to aircraft sequencing problem," Flow Control of Congested Networks. Vol. 38, Springer-Verlag, Berlin, 1987, pp. 323-339.

${ }^{8}$ Dear, R. G., and Sherif, Y. S. "Dynamic scheduling of aircraft in high density terminal areas," Microelectronics Reliability Vol. 29, No. 5, 1989, pp. 743-749

${ }^{9}$ Balakrishnan, H., and Chandran, B. "Scheduling Aircraft Landings under constrained position shifting," AIAA Guidance, Navigation, and Control Conference, Keystone, CO, United states, August 21, 2006 - August 24, 2006.

${ }^{10}$ Balakrishnan, H., and Chandran, B. "Efficient and Equitable Departure Scheduling in Real-time: New Approaches to Old Problems," 7th USA - Europe Air Traffic Management Research and Development Seminar, Barcelona, Spain, July 02-05, 2007.

${ }^{11}$ Rathinam, S., Wood, Z., Sridhar, B., and Jung, Y. C. "A Generalized Dynamic Programming Approach for a Departure Scheduling Problem," AIAA Guidance, Navigation, and Control Conference, Chicago, Illinois, August 10-13, 2009.

${ }^{12}$ Gupta, G., Malik, W., and Jung, Y. "Incorporating Active Runway Crossings in Airport Departure Scheduling," AIAA Guidance, Navigation and Control Conference, Toronto, Canada, August 2-5, 2010.

${ }^{13}$ Solveling, G., Clarke, J. P., Johnson, E. L., and Solak, S. "Runway Operations Optimization in the Presence of Uncertainties," 10th AIAA Aviation Technology, Integration, and Operations (ATIO) Conference. AIAA, Fort Worth, Texas, USA, 2010. 